Prototipos de vivienda de madera de baja huella ecológica y alta eficiencia energética para su aplicación en modelos edificatorios en América Latina Luis Velasco Roldan, Lenin Abatta Jácome, Ángel Hevia Acuña

\title{
Prototipos de vivienda de madera de baja huella ecológica y alta eficiencia energética para su aplicación en modelos edificatorios en América Latina
}

\section{High efficiency and low footprint wooden housing prototypes \\ for Latin America}

\begin{abstract}
Luis Velasco Roldan*
Grupo de Investigación en Energías Renovables. Departamento de Ingeniería Mecánica y Energía integrado en la Universidad de las Fuerzas Armadas, Quito (Ecuador) luisvelascoroldan@gmail.com

Lenin Abatta Jácome**

Grupo de Investigación en Energías Renovables. Departamento de Ingeniería Mecánica y Energía integrado en la Universidad de las Fuerzas Armadas, Quito (Ecuador)

Irabatta@espe.edu.ec

Angel Hevia Antuña***

Grupo de Investigación en Energías Renovables. Departamento de Ingeniería Mecánica y Energía integrado en la Universidad de las Fuerzas Armadas, Quito (Ecuador)

angelheviaa@gmail.com
\end{abstract}

\footnotetext{
* Arquitecto por la Escuela Técnica Superior de Arquitectura del Vallés, Universidad Politécnica de Cataluña (1999). Doctorado en Arquitectura y Eficiencia Energética en Edificios por el departamento de Construcciones Arquitectónicas de la Escuela Técnica Superior de Arquitectura de Barcelona, Universidad Politécnica de Cataluña. Ejercicio libre de la profesión desde Junio del 2000. Técnico asesor responsable de las áreas de construcción e instalaciones del Área Técnica del Colegio Oficial del Arquitectos de Baleares, España, desde junio 2004 . Palma de Mallorca, España. Investigador de la Universidad de las Fuerzas Armadas (ESPE) dentro del Departamento de Ingeniería Mecánica y Energía integrado en el Grupo de Investigación en Energías Renovables. Quito, Ecuador (2013/2016)

** Ingeniero Mecánico por la Universidad de las Fuerzas Armadas ESPE, Máster en Estructuras por la Escuela Politécnica Nacional, Ecuador. Docente del Departamento de Ciencias de la Energía y Mecánica de la Universidad de las Fuerzas Armadas ESPE. Investigador del Grupo de Investigación en Energías Renovablesadscrito al Departamento de Ingeniería Mecánica y Energía. Quito, Ecuador (2013/2016).
}

***Arquitecto. Cursa sus estudios en la Escuela Técnica Superior de Arquitectura de Madrid, Universidad Politécnica de Madrid, entre 1972 y 1985, donde fue alumno de J. M. López-Peláez. Participa a finales de los años 70 en la revista El ecologista y durante los años 90 en la revista D'A. Ejercicio libre de la profesión desde En 1987 realizando trabajos de arquitectura, construcción y paisaje. 


\section{Resumen}

El abandono de técnicas constructivas tradicionales y la sustitución de estas por materiales industrializados de bajas prestaciones ha sustituido en apenas unas décadas los conocimientos constructivos evolucionados durante siglos y perfectamente adaptados a la realidad climática, constructiva, económica y social de su emplazamiento, por modelos inhabitables de un altísima huella ecológica. Con el objetivo de revertir esta tendencia se ha realizado una investigación, centrada en el ámbito ecuatoriano, sobre elementos constructivos, materiales, tecnologías y sistemas que incrementen al máximo la sostenibilidad de la edificación. La investigación se centra en torno a la aplicación de aislamientos térmicos naturales, sistemas de captación solar por efecto invernadero, fachadas ventiladas y cubiertas vegetales. La monitoreo del modelo construido avala las estrategias de eficiencia energética implementadas al obtenerse temperaturas muy estables siempre dentro de la envolvente de confort $\left(20-21^{\circ} \mathrm{C}\right)$, mientras las edificaciones del entorno sufren sobrecalentamientos o requieren de sistemas de calefacción convencionales.

Palabras claves: Arquitectura sustentable Cubierta vegetal, Aislacióntérmica, Madera.

\section{Abstract}

The abandonment of traditional construction techniques and the replacement of these by industrialized low-performance materials has replaced in just a few decades the constructive knowledge developed over the centuries perfectly adapted to the climatic, constructive, economic and social reality of the site by uninhabitable models of a very high footprint ecological With the aim of reversing this trend, research has been carried out, focused on the Ecuadorian environment, on constructive elements, materials, technologies and systems that maximize the sustainability of the building. The research focuses on the application of natural thermal insulation, solar capture systems by greenhouse effect, ventilated facades and green roofs. The monitoring of the built model supports the energy efficiency strategies implemented to obtain very stable temperatures always within the comfort envelope $\left(20-21^{\circ} \mathrm{C}\right)$, while the surrounding buildings suffer overheating or require conventional heating systems.

Keywords: sustainable architecture, green roof, thermal insulation, wood. 


\section{Introducción}

La realidad construida latinoamericana demuestra que gran parte de las construcciones, incluso las situadas en climas templados, presentan importantes problemas de disconfort térmico, fruto de incorrectas estrategias de proyecto y planteamiento constructivo. Es por ello imprescindible el desarrollo de alternativas, construidas en base a la evolución de los conocimientos acumulados durante siglos, evolucionados con el objetivo de proponer modelos edificatorios económica, social y energéticamente sustentables.
Con dicho objetivo se han desarrollado tres proyectos de edificio, válidos para el ámbito climático ecuatoriano, con la mínima huella ecológica y mayor cota de eficiencia energética. Concretamente el proyecto desarrolla la aplicación de aislantes térmicos naturales, una cubierta vegetal de bajo coste y un sistema de fachada ventilada, así como un sistema de captación de energía solar por efecto invernadero adaptado a latitudes ecuatoriales. El resultado de dicha investigación son los tres proyectos identificados como E1 (figura 1), E2 (figura 2) y E3 (figura 3) y

Figura 1 y 2: Plantas de los modelos E1 y E2. El área amarilla indica la superficie de captación solar propuesta en cubierta.

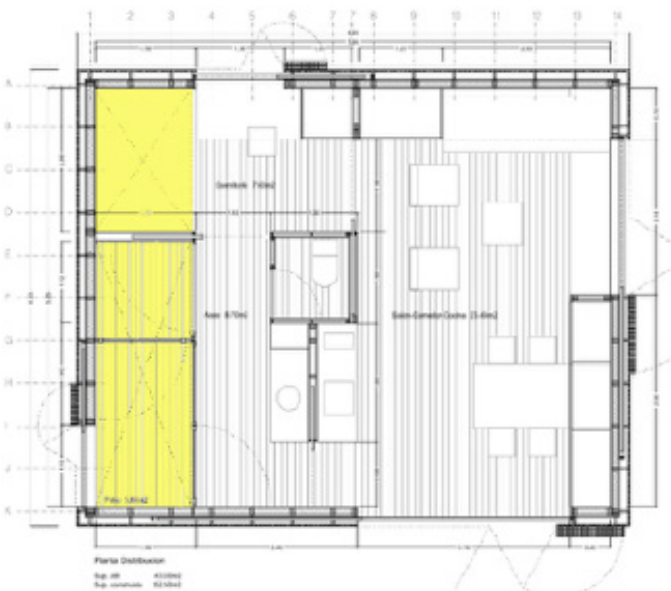

Fuente: elaboración propia

Figura 3: Sección constructiva y alzados del prototipo E3

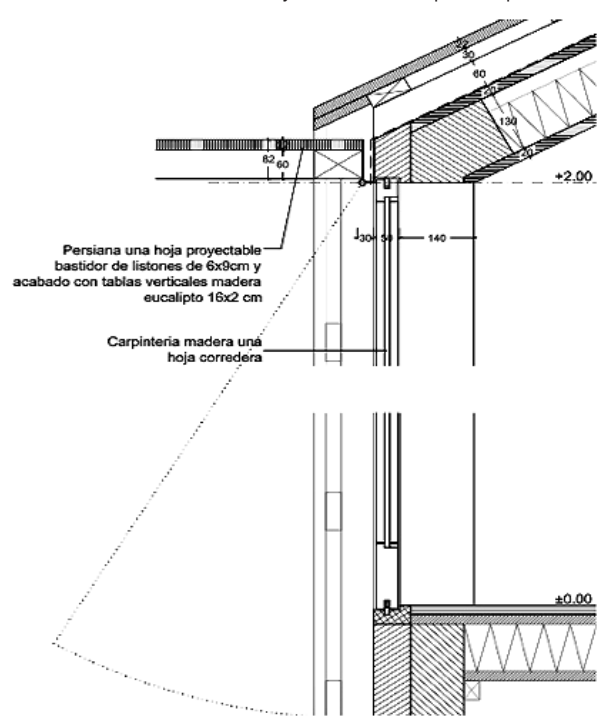

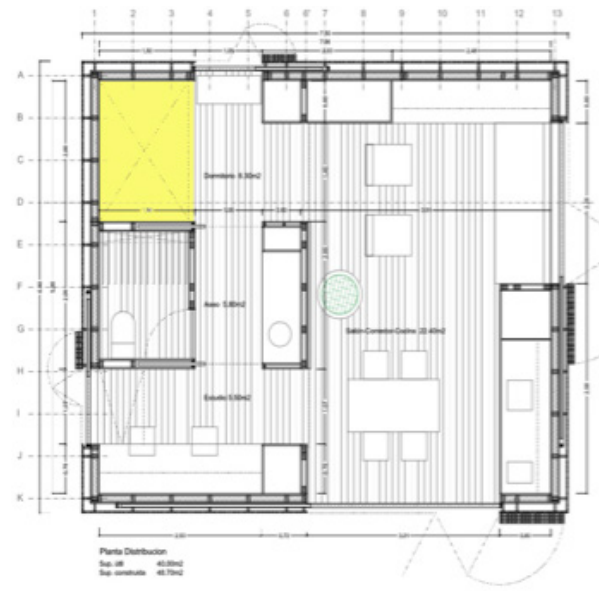
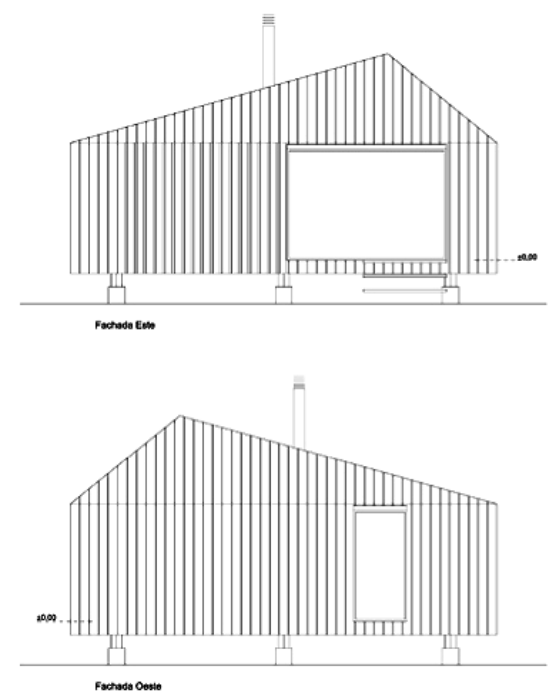

Fuente: elaboración propia 
Tabla 1: Conductividades de las distintas biomasas ensayadas

\begin{tabular}{|l|l|l|}
\hline & Densidad aparente(gr/cm3) & Conductividad térmica (W/0K.m2) \\
\hline Raquis de palma en panel & 0,1825 & 0,0867 \\
\hline Raquis de palma a granel & 0,1483 & 0,0613 \\
\hline Bagazo de caña de azúcar & $0,0625-0,0781$ & $0,0255-0,034$ \\
\hline Cascarilla de cacao & 0,0789 & 0,0215 \\
\hline Virutas guadua. Sección 2-3 cm & 0,0785 & 0,0389 \\
\hline Totora granel. Longitud: $1-2 \mathrm{~cm}$ & 0,0785 & 0,0321 \\
\hline
\end{tabular}

Fuente: Elaboración propia

la construcción y monitoreo de uno de ellos, el prototipo E2.

Los proyectos cuentan con distintas estrategias de eficiencia energética, pues cada uno de ellos ha sido diseñado para responder a los principales climas del país. De esta forma, el modelo E1 está diseñado para climas fríos de la sierra, por lo que la superficie de captación solar y el aislamiento térmico de envolvente se refuerza con respecto al prototipo E2, diseñado para climas templados. El prototipo E3, de distribución similar al modelo E1, se ha diseñado para climas costeros y amazónicos. El prototipo E3, por lo tanto, no desarrolla estrategias de captación solar y sí una especial atención a la ventilación y protección solar de todo el edificio.

En toda América Latina, las actividades agrícolas generan muy diversos residuos no valorizados que podrían convertirse en componente fundamental para muy diversos materiales de construcción (Beraldo A.L. (UPV), 2011). Muchos de estos componentes cuentan con una gran capacidad aislante que podría mejorar las características térmicas de los sistemas constructivos tradicionales y contemporáneos. En torno a dicho efecto aislante se encuentran estudios iniciales de ciertas biomasas, como la cascarilla de arroz o café (Salazar, García y Olaya, n.d.) (Salas, Veras y Castro, 2012) (Beraldo A.L, 2011).

Compuestos encaminados a determinar las posibilidades de inclusión de los residuos de biomasa generados por los cultivos propios de cada área de Ecuador en elementos constructivos tales como: bloques, adobes, tapial o pisos de tierra estabilizados, paneles, mantas, etc. (Velasco, Delgado y Goyos, n.d.) se encuentran en fase final de evaluación por parte del Grupo de Energías Renovables del Departamento de Ciencias de la Energía y Mecánica en su carrera de Ingeniería Mecánica de la Universidad de las Fuerzas Armadas - ESPE con resultados muy prometedores por sus bajas conductividades. Los resultados preliminares se resumen en la siguiente tabla (tabla 1 ).

\section{Situación: ámbito climático y edificatorio.}

La región de la Sierra ecuatoriana está atravesada por la cordillera de los Andes, que recorre el país de Norte a Sur. Se divide en dos sistemas paralelos que crean una serie de microclimas conocidos como escalones climáticos (INAMHI, n.d.). En el tropical andino la temperatura media varía entre los 20 y $25^{\circ} \mathrm{C}$. Las lluvias son escasas y la atmósfera seca. Comprende todas las tierras bajas hasta una altura de 1500 m. El subtropical andino va desde los 1500 hasta los $2500 \mathrm{~m}$, con una temperatura media de $20{ }^{\circ} \mathrm{C}$. Lluvias abundantes en invierno y poco frecuentes en verano lo caracterizan. El clima templado, con una temperatura media de $17^{\circ} \mathrm{C}$, se sitúa en los lugares que van desde los 2500 hasta los $3500 \mathrm{~m}$ de altitud. Se caracteriza este escalón climático por tener lluvias abundantes, granizadas frecuentes y ambiente nublado. Es la franja climática más poblada de la Sierra. El piso frío comprende todos aquellos lugares que van desde los 3500 hasta las $5650 \mathrm{~m}$. Su temperatura varia entre 1 y $10^{\circ} \mathrm{C}$. Se dan en él 
aguaceros torrenciales, neblinas espesas y lloviznas casi constantes.

El régimen de temperaturas diarias es relativamente estable durante todo el año en toda la sierra. En el gráfico (figura 4) se observa como toda en todas las provincias de la región, las bajas temperaturas exigen la presencia de aislamiento térmico junto con algún tipo de sistema de calefacción. Por el contrario, no existen en la sierra problemas de altas temperaturas, a excepción de la provincia de Loja. Si existen en cambio problemas puntuales derivados de la alta radiación en donde se hará necesario el control de la radiación solar.

Las edificaciones tradicionales se ubican por lo general en terrenos llanos o laderas orientadas a Sur en busca de un mayor asoleo. En su mayor parte se caracterizan por muros de piedra o tierra (tapial o adobe), pisos de tierra y cubiertas de paja o teja sobre estructura de madera. En zonas menos frías se utiliza con frecuencia el bahareque, que consiste en muros de cerramiento formados por una estructura de madera o cañas recubierta de barro y paja.

La construcción en tierra es un recurso constructivo de bajo impacto ambiental, acceso local e integrado en las tradiciones constructivas locales, pero es un material con un alto coeficiente de conductividad, por lo que resulta imposible alcanzar las temperaturas de confort interior sin altísimos consumos energéticos. En las zonas altas de los páramos se mantiene un tipo de choza mixta de muros de tierra y tejado de paja que se extiende prácticamente hasta el suelo. La gran capacidad aislante de la paja protege el interior de las bajas temperaturas y de los vientos fríos. En todos los tipos constructivos los suelos apisonados constituyen un foco frío.

El Valle de los Chillos (Ecuador), emplazamiento inicial del prototipo E2, goza de un clima suave con fuerte radiación (5-5,5 Kwh/m2) combinada con una imprevisible nubosidad. Las temperaturas durante el día pueden superar los $20^{\circ} \mathrm{C}$ pero descienden por la noche hasta los $12^{\circ} \mathrm{C}$ y

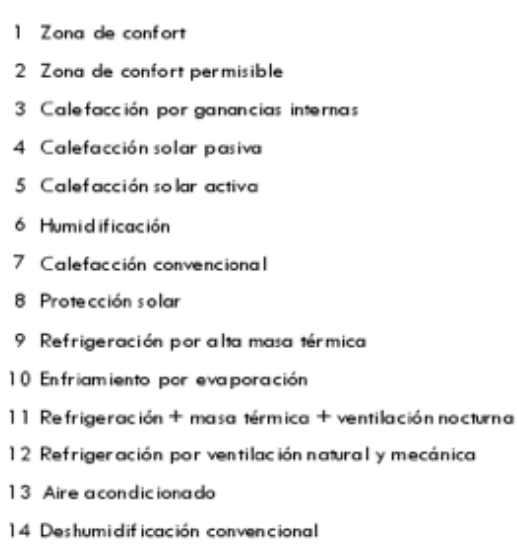


son estables durante todo el año. Los edificios del entorno edificado del campus universitario, pese a lo moderado del clima, sufren problemas de sobrecalentamiento o de temperaturas interiores bajas.

\section{Casos de estudio}

Los tres prototipos desarrollados cuentan con una cámara de entre 13 y 15 cm de espesor (confinada entre paneles de madera) que envuelve totalmente el espacio interior. Se pretende rellenar este intersticio en cada uno de los edificios con: raquis de palma (E1), piedra pómez (E2), y pajonal (E3). Las biomasas ensayadas se han seleccionado entre aquellas existentes en las regiones de implantación de los prototipos.

Por otro lado, la capacidad de las fachadas ventiladas de implementar estrategias de aho- rro energético se encuentran ampliamente estudiadas y ensayadas (Heine, Leandro, 2002), pese a que su aplicación en muchos caso se ha fundamentado en razones meramente estéticas. La estrategia de incorporar al diseño una doble piel en latitudes ecuatoriales tiene dos importantes funciones. En climas cálidos actúa a modo de protección solar de toda la envolvente interior. En climas de montaña reduce la velocidad con la que inciden los vientos fríos sobre los muros aislados, reduciendo en gran medida el coeficiente convección y con ello las pérdidas de calor. Por otro lado la radiación fría espacial no incide sobre los muros aislados reduciendo pérdidas energéticas durante la noche. con el objetivo de ensayar su comportamiento en clima moderado, frío o cálido la doble piel ha sido planteada en los tres prototipos (figura 5).

La sustitución de las cubiertas tradicionales por planchas de fibrocemento, chapa de acero

Figura 5: Sección constructiva del modelo E3 en el que esta doble piel ventilada se extiende por toda la cubierta

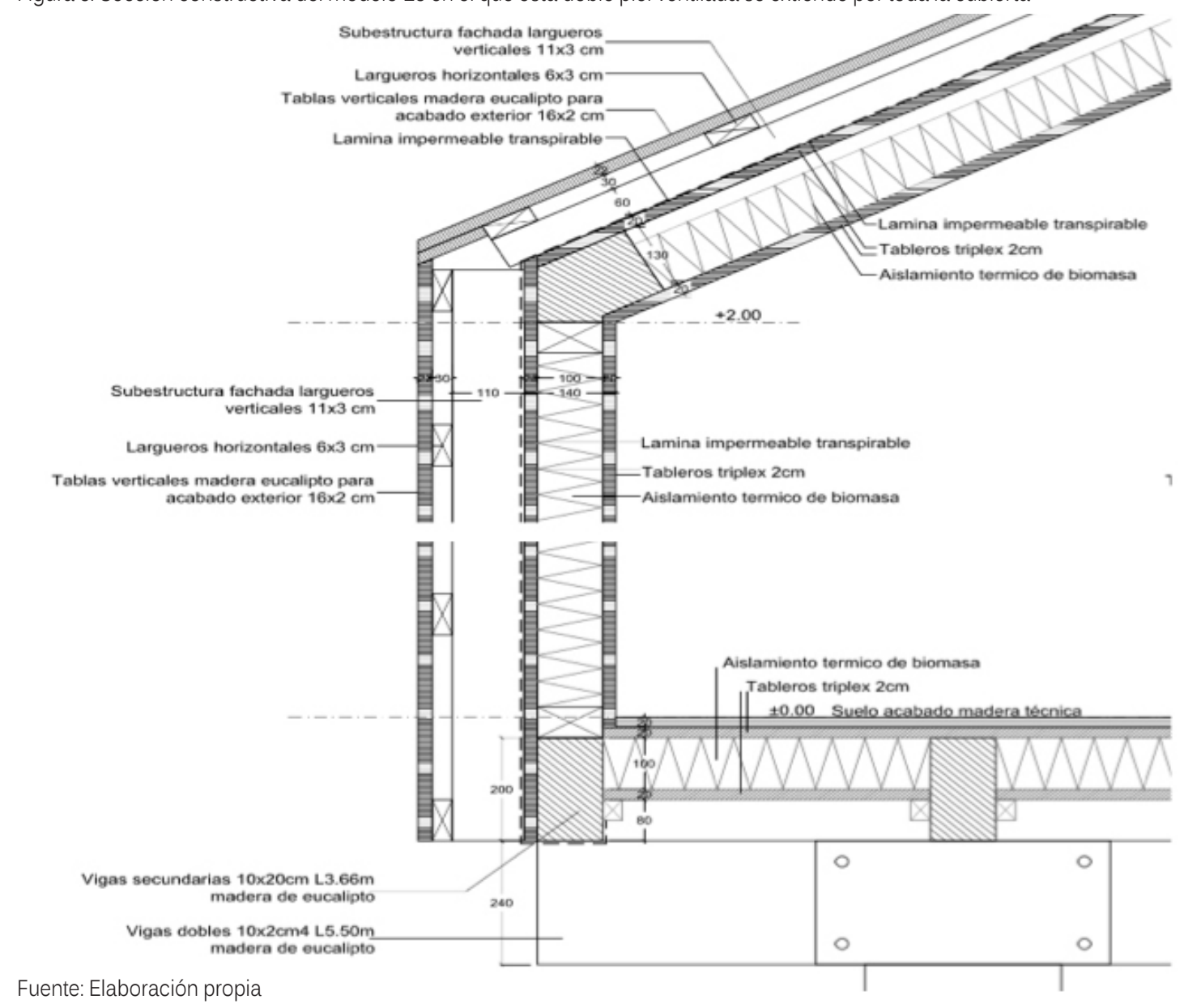


galvanizado o losas de hormigón supone un empeoramiento radical de la sostenibilidad edificatoria al utilizarse materiales de altísima huella ecológica, no reciclables y procedentes de zonas lejanas. La eficiencia energética de las edificaciones vernáculas, lejos de mejorarse, empeora radicalmente, pues las altísimas temperaturas que provoca la radiación solar por el acceso indiscriminado de energía a través de la cubierta provoca graves problemas de confort térmico interior. Se han ensayado hasta el momento cubiertas alternativas (Castañeda, Argüello, \& Vecchia, 2011) (Machado, Brito, \& Neila, 2000) buscando la mejora del aislamiento térmico y cierta inercia térmica que permita una estabilidad térmica en climas fríos. La cubierta vegetal es una de ellas y cuenta con una amplia bibliografía y numerosos casos de estudio en Norte América y Europa. (Neila, Bedoya y Acha, 2008) (Blackhurst, Hendrickson, Asce, Matthews y Asce, 2010). En situación urbana, entre sus beneficios, se encuentran la absorción de $\mathrm{CO} 2$, la mitigación del efecto "isla de calor", la regulación de la humedad ambiental y el retardo en el vertido de las Iluvias torrenciales a la red urbana. En cualquier emplazamiento, la inercia térmica de este tipo de cubiertas permite la protección del interior frente a cambios bruscos de temperatura y radiaciones solares intensas. Se estima que en una cubierta vegetal la radiación incidente es reflejada en un $27 \%$, las plantas y el sustrato absorben el $60 \%$ y únicamente el 13\% de la radiación es transmitido por conducción al interior (Machado, Brito, \& Neila, 2000). En el ámbito ecuatoriano la solución se considera de gran potencial para eliminar el problema de sobrecalentamiento de buena parte de las cubiertas del país en zonas cálidas de alta radiación así como las pérdidas de calor en climas fríos de montaña.

En latitudes cercanas al Ecuador la radiación solar sobre las aberturas de la fachada norte y sur incide con ángulos entre 67 y 90 dificultando el acceso solar al interior del edificio y por lo tanto la captación por efecto invernadero. En las fachadas este y oeste, la potencia de la radiación incidente es limitada debido al reducido ángulo solar. Dicha circunstancia obliga a que estrategias de captación en latitudes cercanas al Ecuador deban ser distintas a las estrategias habituales en latitudes meridionales, basadas en la captación en invierno de la radiación sobre las fachadas norte o sur (dependiendo del hemisferio en el que se sitúen). En latitudes ecuatoriales los elementos de captación solar deberían situarse preferentemente en la cubierta del edificio, en dónde los rayos solares, al incidir de forma casi perpendicular al vidrio, limitan las reflexiones hacia el exterior e inciden sobre una gran superficie interior. Pequeñas superficies vidriadas situadas en la cubierta, por lo tanto, pueden aportar la energía necesaria para la calefacción interior en climas templados y fríos si son controladas las pérdidas de calor durante la noche o el exceso de radiación durante el día en dichos elementos.

El prototipo E3, dada su ubicación en clima cálido prescinde de estrategias de captación solar. Los otros dos prototipos están dotados de una cubierta vidriada horizontal (vidrio doble laminado) de superficie proporcionada a las necesidades de calefacción. En el caso del prototipo E1, esta cuenta con 9,5 m2 y se sitúa sobre el patio invernadero, la ducha y la habitación (ver zona amarilla en figura 1). El prototipo E2 cuenta con 3,5 m2 situados sobre la habitación.

Por último citar que es objetivo principal de la investigación el monitoreo del comportamiento térmico de edificios construidos para su argumentación y difusión como modelos arquitectónicos "alternativos". Existen muy pocas experiencias de monitoreo en América del Sur que evalúe convenientemente las estrategias de ahorro energético ensayadas en proyectos construidos. (Alamino, Kuchen, Gil Rostol y Alonso Frank, 2015)(C. Filippín, 2004). El análisis propuesto se basa en la toma continua de valores de temperatura del aire (interior y exterior) y humedad relativa, complementadas por una serie de mediciones puntuales de la temperatura radiante de los paramentos. Se pretende testar un patrón real de uso de la vivienda por lo que el monitoreo se realzará con la vivienda habitada. Los valores de temperatura superficial de los paramentos de la envolvente térmica se han realizado, por motivos comparativos entre los edificios del entorno sin considerar su patrón de uso al considerase este irrelevante 


\section{Análisis del modelo construido y monitorizado prototipo E2}

El prototipo se sitúa en la Universidad de las Fuerzas Armadas ESPE (figura 6 y 7) y pretende alcanzar la mínima huella ecológica mediante el estudio de los recursos materiales y los mínimos requerimientos energéticos. Una vez finalizada su vida útil será posible la reutilización de sus partes o su reciclaje. Puede observarse en la figura 1 la distribución interior del prototipo, de 45 metros cuadrados útiles, que alberga un programa convencional de vivienda (sala de estar, comedor, cocina, habitación y estudio) de planta abierta, para asegurar la difusión de la energía captada por el invernadero.

\section{Sistema constructivo.}

El prototipo E2 demuestra que, pese a construirse con un sistema constructivo ligero, es posible alcanzar temperaturas de confort sin necesidad de sistemas de climatización artificial. Esto ha sido posible gracias a un aislamiento térmico capaz de reducir considerablemente las pérdidas de energía y una inercia térmica capaz de almacenar la energía captada durante el día por efecto invernadero, independientemente de las variaciones climáticas diarias y el número de horas de radiación recibidas.

La cimentación está formada por zapatas de hormigón ciclópeo para el aprovechamiento de material pétreo local. Desde las zapatas

Figuras 6 y 7: Fotografías del prototipo E2
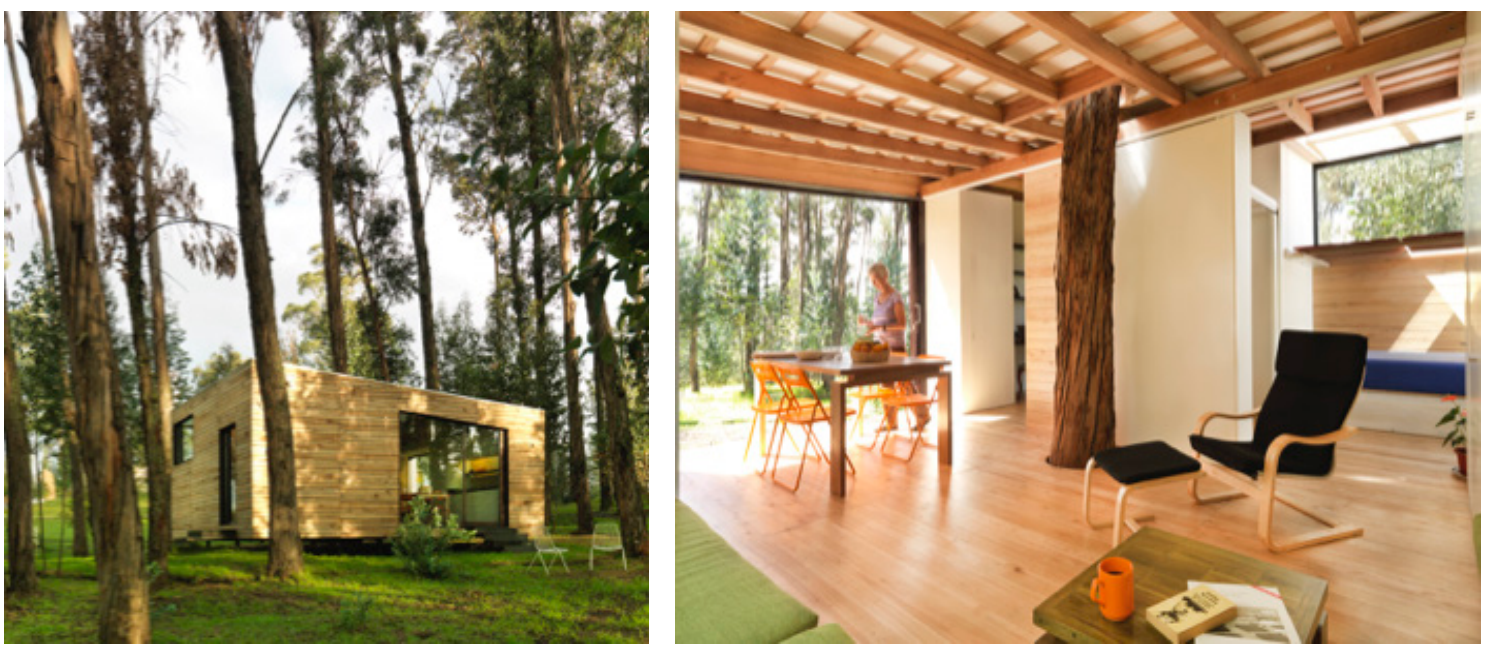

Fuente: elaboración propia

Figura 8 y 9: Estructura del edificio
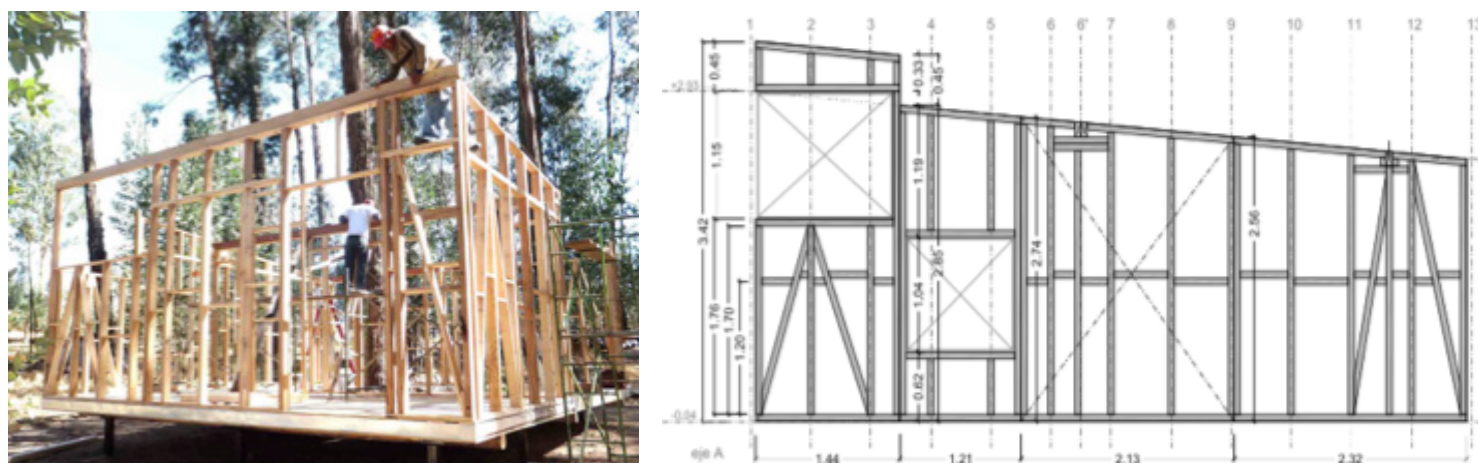

Fuente: elaboración propia 
arrancan 12 perfiles metálicos hasta las vigas principales del suelo. Todo el resto de estructura se ha realizado con madera de eucalipto por su alta resistencia, bajo costo y rápido crecimiento. Los muros están constituidos por montantes de 10x6 cm. cada $60 \mathrm{~cm}$. formando bastidores reforzados mediante travesaños horizontales y diagonales.

Esta estructura de luces cortas permite el uso de escuadrías pequeñas y un mayor aprovechamiento del árbol. Dada la alta sismicidad de la zona, el conjunto de bastidores se refuerza puntualmente por cruces de San Andrés de chapa de acero de $1,5 \mathrm{~mm}$ y $10 \mathrm{~cm}$. de anchura. Los forjados, formados por vigas de madera, se recubren inferior y superiormente con tableros de madera contrachapada de $15 \mathrm{~mm}$, fuertemente atornillados para la colaboración de estos en la transmisión de esfuerzos (figura 8 y 9).

El interior de los bastidores de las paredes y entrevigado del suelo, de 13 y $15 \mathrm{~cm}$ de espesor respectivamente, se rellena de piedra pómez, quedando esta confinada por tableros contrachapados de $6 \mathrm{~mm}$ de espesor. La piedra pómez será la encargada de aportar el necesario aislamiento e inercia térmica a la edificación (figura 10).

El edificio incluye una cubierta vegetal consistente en una capa de humus $(7 \mathrm{~cm}$.) sobre material drenante y aislante de piedra pómez $(10 \mathrm{~cm}$.) envuelto en fieltro geotéxtil. Ambas capas se superponen a la doble impermeabilización asfáltica y la estructura de madera de la cubierta de reducida inclinación (6\%) (figura 11 y 12).

El sistema de fachada consiste en un revestimiento de tablas de madera local (laurel) separadas entre ellas $5 \mathrm{~mm}$, y fijas a la pared interior aislada mediante chapa de galvanizado doblada en forma de Z de $12 \mathrm{~cm}$ de sección. De esta forma se obtiene una cámara de aire ventilada a modo de fachada pluvial que evita

Figura 10: Aislamiento térmico de piedra pómez en pisos y paredes

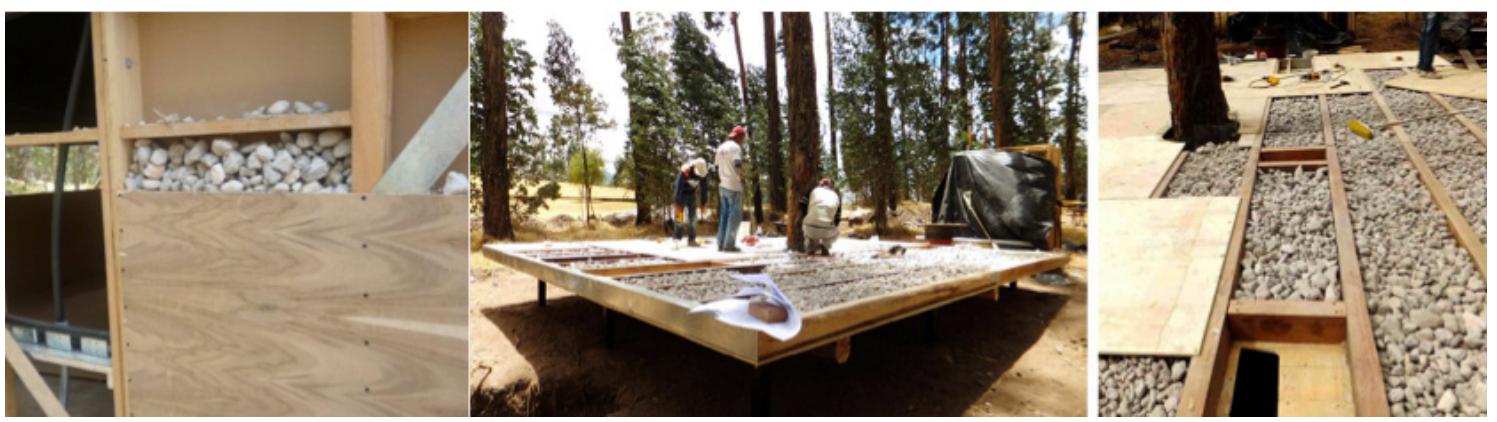

Fuente: elaboración propia

Figura 11 y 12: Cubierta vegetal y fachada ventilada
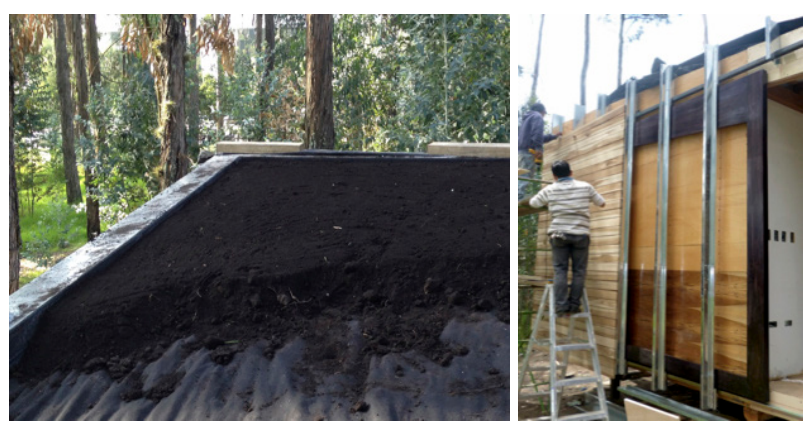

Fuente: elaboración propia 
el humedecimiento de la fachada interior (que por lo tanto no requiere impermeabilización y se mantiene transpirable). La cámara de aire permite el secado perimetral de la tabla de madera exterior, reduciendo el riesgo de proliferación de hongos o pudrición de esta.

La captación solar necesaria para el calentamiento de la vivienda se obtiene mediante el diseño de un invernadero cuya situación puede observarse en la figura 13. Este se encuentra vidriado en dos de sus fachadas y su parte superior. Los tableros de la litera superior interceptan los rayos solares procedentes de múltiples orientaciones. El invernadero puede cerrarse en su perímetro y reducir de este modo las pérdidas de energía durante la noche provocadas por la radiación fría (espacial) y las bajas temperaturas (figura 13 y 14).

\section{Medición comparada de las temperaturas su- perficiales}

Para el análisis de la temperatura superficial de la cubierta y fachada del edificio se ha utilizado una cámara Fluke Ti25 dotada con corrector de emisividad, con una sensibilidad térmica de $0.1^{\circ} \mathrm{C}$ a $30^{\circ} \mathrm{C}$ para un rango de temperaturas de entre -20 y $350^{\circ} \mathrm{C}$ y una precisión de $2^{\circ} \mathrm{C}$ o el $2 \%$. Se han realizado series de mediciones puntuales durante las horas de mayor y menor temperatura exterior, así como en periodos de máxima y mínima insolación diurna.

En climas de montaña de alta radiación el intercambio de energía entre el interior y el exterior no solo depende del diferencial de temperaturas entre el aire interior y el exterior, pues la radiación solar calienta intensamente

Figura 13 y 14: Invernadero
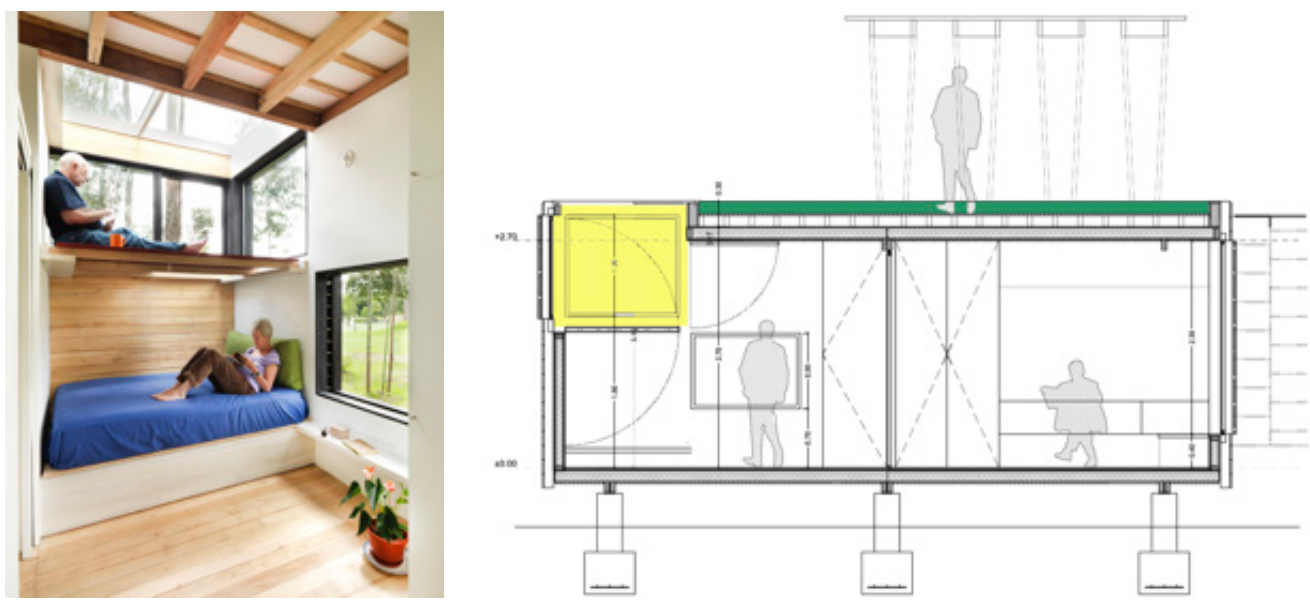

Fuente: elaboración propia

Figura 15-17: Termografías de distintos edificios del Campus ESPE

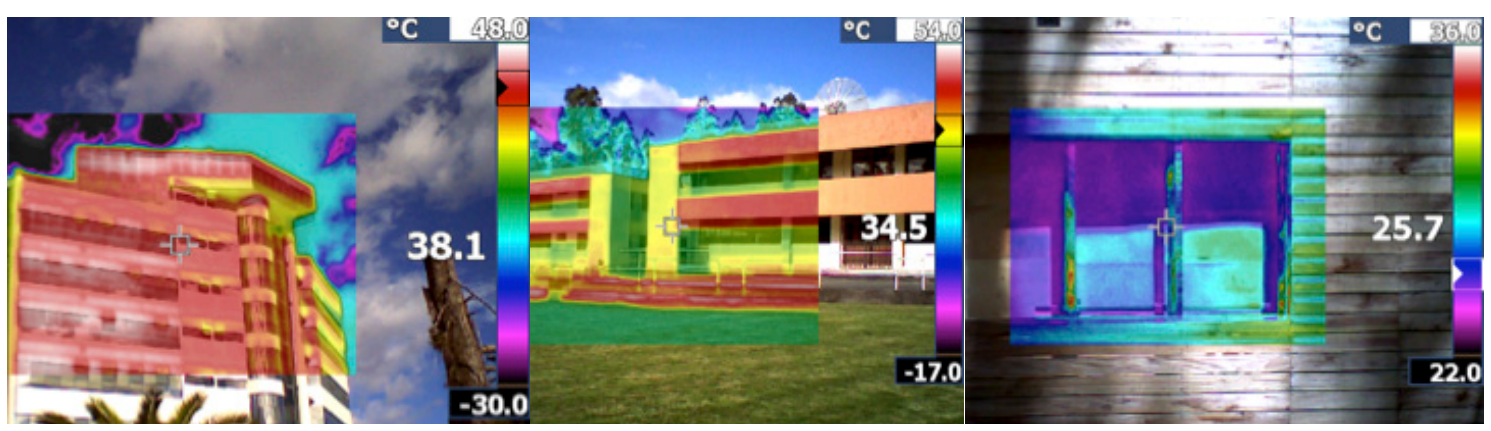

Fuente: elaboración propia 
las superficies de la envolvente térmica durante el día, mientras que la radiación espacial las enfría durante la noche. En el caso del prototipo E1 el análisis termográfico de la envolvente térmica demuestra como la fachada ventilada y la cubierta vegetal atemperan el perímetro aislado del prototipo. Si observamos las figuras (figura15,16 y 17) se observan los problemas de sobrecalentamiento del los edificios del entorno que alcanzan al atardecer temperaturas de hasta $45^{\circ} \mathrm{C}$, mientras que la cara exterior de la fachada aislada del prototipo E2, protegida por las lamas de madera, se mantiene a $22^{\circ} \mathrm{C}$.

Del mismo modo, mediante las termografías, es posible evaluar las temperaturas superficiales

Figura 18: Termografía del prototipo E1

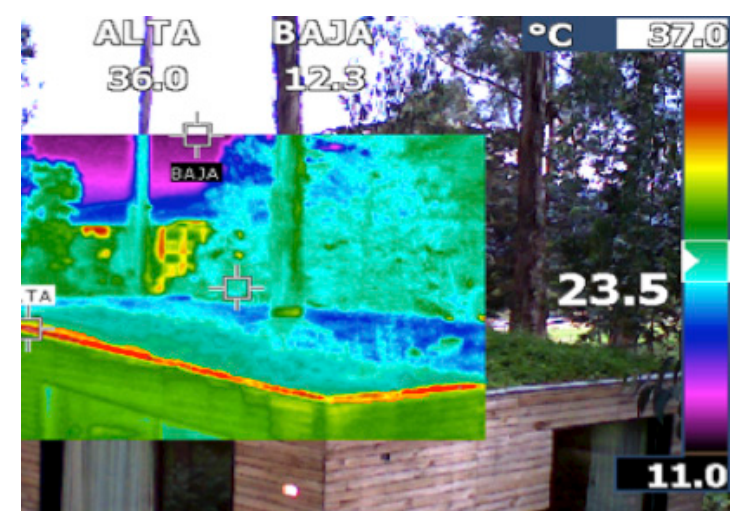

Fuente: elaboración propia de la cubierta vegetal en comparación con las que podrían obtenerse con una cubierta de chapa de acero en un día soleado. Mientras la superficie vegetal se mantiene en torno a los $22^{\circ} \mathrm{C}$, puede observarse como la chapa perimetral que protege de la lluvia la cámara de aire alcanza temperaturas en torno a $35^{\circ} \mathrm{C}$ (temperatura que alcanzaría una cubierta de chapa convencional). La cara exterior de la fachada ventilada alcanza lo $30^{\circ} \mathrm{C}$ mientras la cara interior se mantiene a $22^{\circ} \mathrm{C}$ (figura 18).

Por otro lado, el monitoreo de las temperaturas interiores demuestran una gran estabilidad de temperaturas de la vivienda, solo alteradas por la abertura inferior y superior del árbol y los perímetros de las carpinterías, en donde la correderas se conforman como los únicos puntos de infiltración de aire y puentes térmicos de la vivienda, responsables del mínimo descenso interior de las temperaturas durante la noche. Pese a ello, dada la ausencia de presión de viento en la zona, este no representa un problema térmico grave.

\section{Monitorización térmica interior del prototipo E2}

Para el análisis empírico de las distintas hipótesis de funcionamiento es indispensable registrar de una manera precisa y continua las variables de temperaturas interior y exterior así

Figura 19: Régimen diario de temperatura del prototipo E1

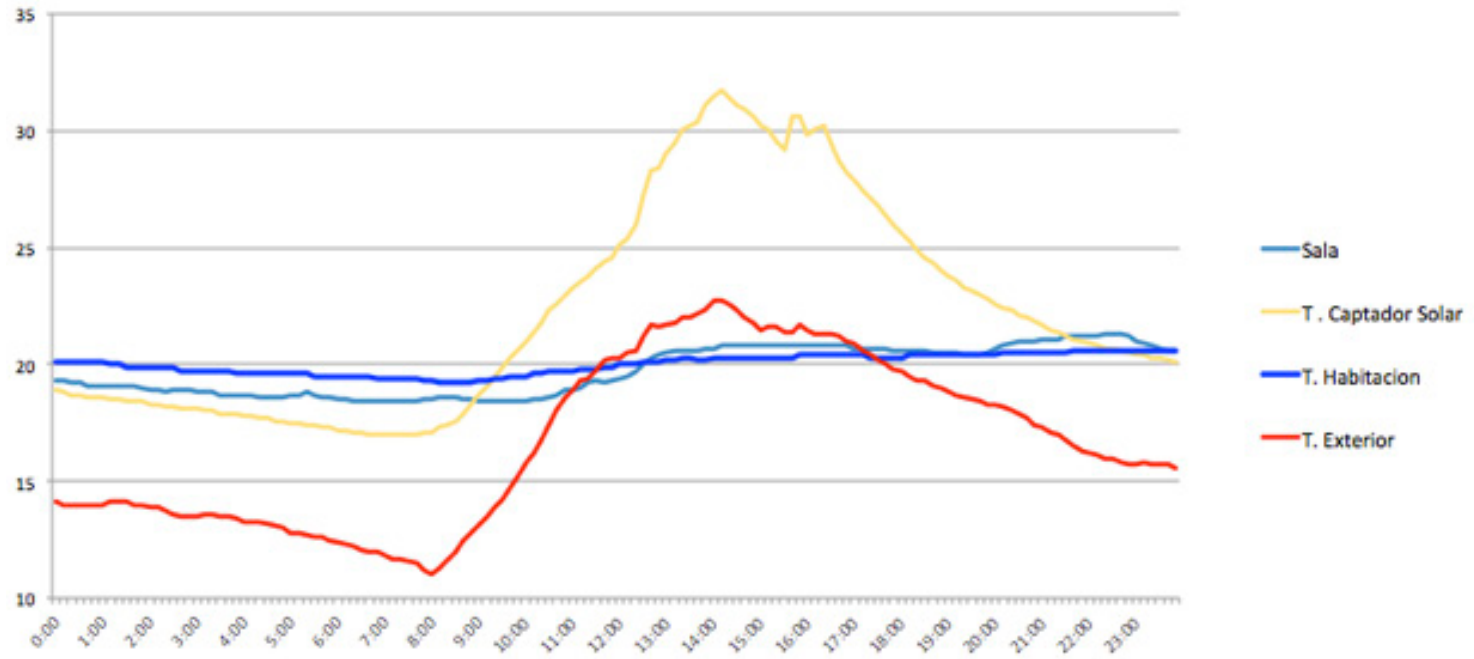

Fuente: elaboración propia 
como la humedad relativa. El sistema Testo Savieris permite la toma y almacenamiento de valores de temperatura y humedad, así como la interpretación gráfica inmediata de los datos recibidos. Los aparatos de medición continua complementarios empleados son data loggers Testo con un rango de medición de entre -35 y $70^{\circ} \mathrm{C}$ y una precisión de $\pm 0,5$ ${ }^{\circ} \mathrm{C}\left(-35 \ldots+55^{\circ} \mathrm{C}\right)$. Todos los aparatos de medición han sido colocado en su posición 24 horas antes de inicio de la toma de datos para permitir su aclimatación convenientemente, protegidos de la radiación solar y del agua o el viento para evitar posibles distorsiones. Durante todo el periodo de medición se realizó un diario de anotaciones con referencia a todo lo que pudiera afectar o alterar la medición o la comprensión de esta (figura 19).

Se han dispuesto 3 termómetros para la recogida de datos automáticos en el interior de la vivienda para la toma de temperaturas y humedad. El primero se sitúa en la sala-comedor-cocina, el segundo en la habitación (ambos a una altura de 2 metros) y el tercero en la parte superior de la habitación, principal zona de captación de energía por efecto invernadero, a 3,5 metros de altura. En el análisis de los registros se observa una completa estabilidad de la temperatura interior a lo largo de los días, independientemente del régimen de nubosidad existente. La temperatura del aire en toda la vivienda se sitúa en torno a los $20^{\circ} \mathrm{C}$, demostrando el efecto estabilizador de la inercia térmica aportada por la envolvente de piedra pómez. La variabilidad de la temperatura interior de la vivienda a lo largo del día es de apenas $2^{\circ} \mathrm{C}$. La humedad relativa se mantiene dentro del rango de confort: entre el 55 y el $70 \%$. Se observa una acumulación de calor en el invernadero durante las horas de sol. Esta no supone ningún problema de disconfort al situarse esta en una zona sin la presencia de personas, pero detecta un cierto desaprovechamiento del potencial de energía captada debido a una imperfecta distribución de la energía por convección natural. Podría por lo tanto mejorarse el potencial de captación del invernadero mediante la instalación de un ventilador para forzar una mayor distribución de dicha energía en la vivienda.

Del análisis comparativo de la temperatura interior y exterior se advierte la importancia de la combinación de aislamiento térmico, captación solar e inercia térmica. La temperatura exterior apenas supera la temperatura de confort durante 5 horas al día, lo cual pone de manifiesto la eficiencia del invernadero a la hora de calentar la vivienda. La estabilidad de la temperatura interior demuestra cómo la inercia térmica acumula de una manera conveniente dicha energía disipándola gradualmente a lo largo de la mañana, la tarde y la noche. A su vez, el mantenimiento de temperaturas de confort en el interior, a pesar de las bajas temperaturas nocturnas, demuestra la eficacia del aislamiento térmico aportado por la piedra pómez.

Figura 20 y 21: Edificio de oficinas del rectorado y edificio de oficinas y laboratorios de ingeniería mecánica. ESPE
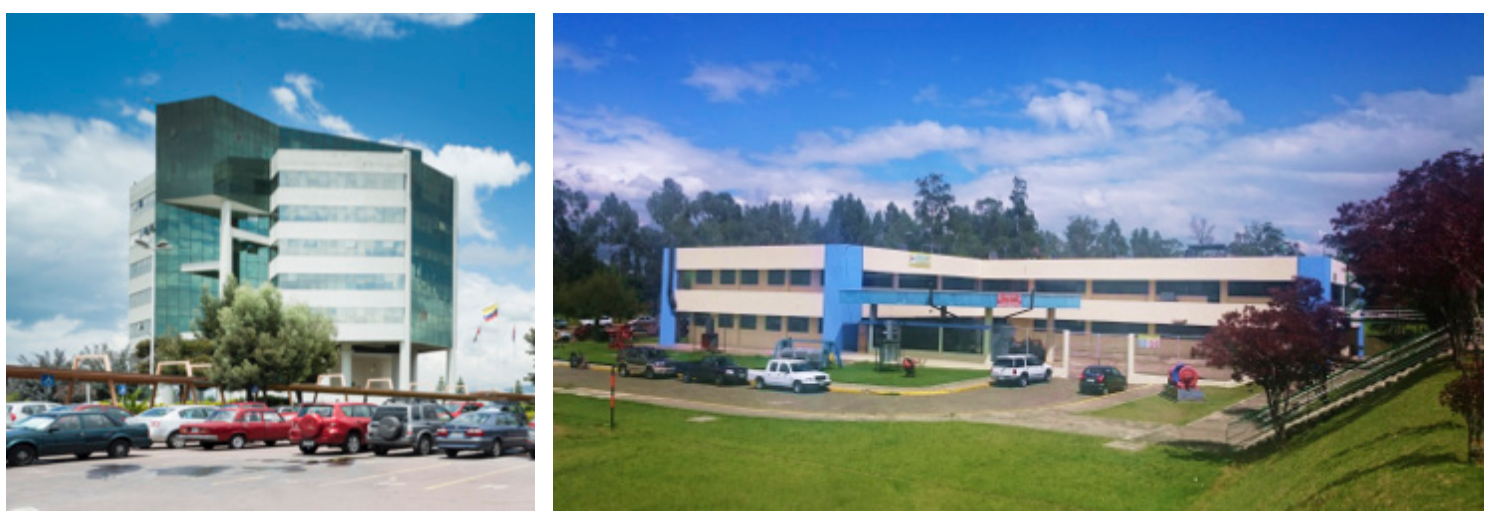

Fuente: elaboración propia 


\section{Monitoreo de las condiciones de temperatura interior}

Para la evaluación comparada del comportamiento del sistema constructivo propuesto, se ha registrado el régimen de temperaturas en el prototipo E2 y en dos oficinas situadas en edificios localizados en el campus universitario con distinto planteamiento constructivo (figura 20 y 21).

Si bien el perfil de uso o las cargas internas de los edificios no es comparable, la selección de estos se ha realizado en función de los tipos constructivos existentes y accesibles en el entorno para su comparación del comportamiento de sus sistemas constructivos sistema constructivos. Los resultados por lo tanto deben tomarse con precaución y complementarse con una toma de datos en edificios de uso residencial próximos en futuras monitorizaciones.

- Oficina del edificio de Rectorado. Orientación Sureste. Limitada por forjados de hormigón y fachadas formadas por un $65 \%$ de vidrio simple tintado (verde oscuro) y un 35\% de bloque de hormigón de $15 \mathrm{~cm}$ de espesor revestido en sus dos caras por mortero de cemento.

- Oficina del edificio de laboratorios de Ingeniería Mecánica. Orientación Este. Planta segunda en edificio de dos alturas con sistema constructivo de losas y pilares de hormigón armado. Ce- rramientos de bloque de hormigón en un 60\% y vidriado en un $40 \%$ mediante carpinterías de acero y vidrios simples. La superficie de vidrio se encuentra protegidas de la radiación solar por unas galerías perimetrales de las que se descuelga un parasol de hormigón.

Los tres espacios presentan comportamientos muy diferentes (figura 22). Mientras el edificio del rectorado presenta periodos de sobrecalentamiento y gran variabilidad de temperaturas interiores provocadas por la ausencia de una inercia térmica y la excesiva captación solar, el edificio de oficinas de los laboratorios del Departamento de Ingeniería Mecánica presenta un comportamiento totalmente distinto. Mantiene temperaturas estables, pero inferiores a las obtenidas en el prototipo E2 y a la temperatura de confort. La gran inercia térmica del sistema constructivo de hormigón mantiene la temperatura estable, pero la ausencia de aislamiento térmico y de energía captada por efecto invernadero provoca temperaturas interiores bajas, fuera de la zona de confort durante todo el día y la noche.

El único espacio que mantiene unas temperaturas estables dentro del a franja de confort es el prototipo E2, demostrando que se trata del sistema constructivo más equilibrado en cuanto a aislamiento, captación solar e inercia térmica para el clima templado en el que se ubica.

Figura 22: Régimen de temperaturas interiores de las tres construcciones

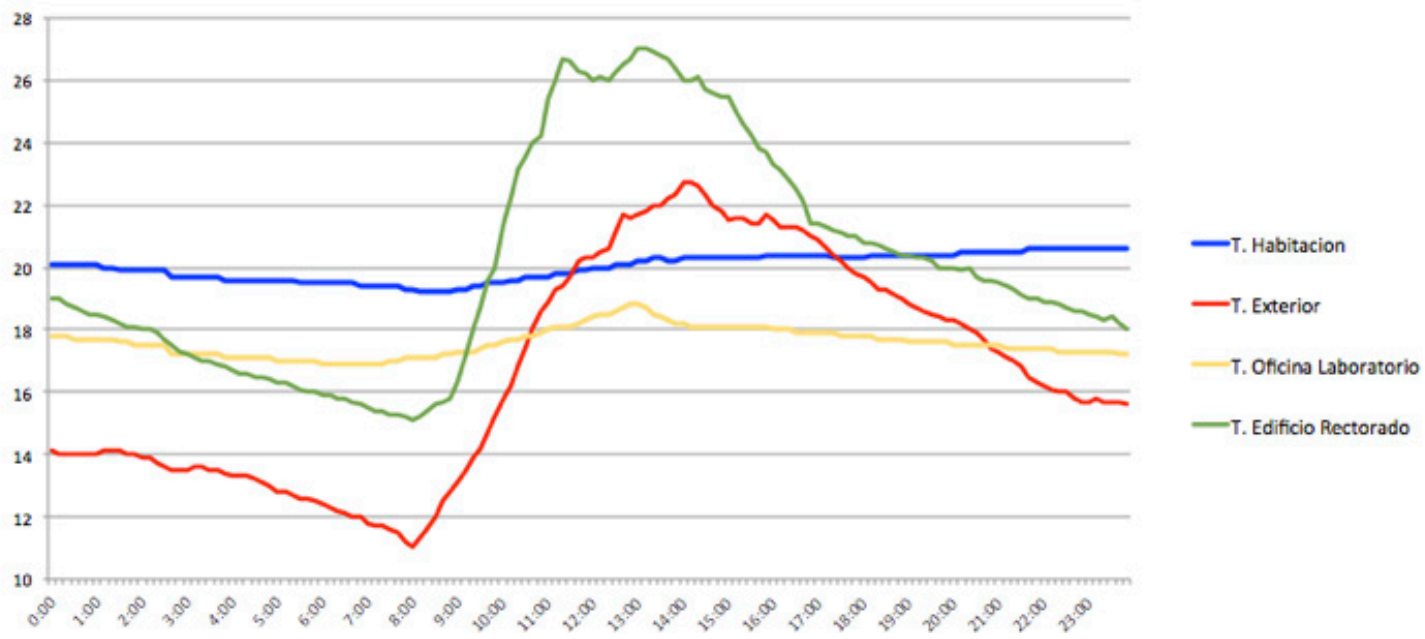

Fuente: Elaboración propia 


\section{Conclusiones}

La construcción y el monitoreo del prototipo E2 aporta por primera vez en el país un ejemplo práctico de comportamiento energético evaluado bajo criterios científicos en torno a soluciones constructivas de alta eficiencia energética de aplicación viable y bajo coste.

Existen en la región recursos naturales aprovechables desde el punto de vista constructivo que pueden permitir una reducción drástica del impacto medioambiental de la construcción, así como una mayor integración paisajística de las edificaciones. Es por ello posible la evolución de los modelos de vivienda bajo un nuevo prisma de sostenibilidad energética, social y económica.
El estudio de la eficiencia energética y la implantación de energías renovables puede ser planeada en clave de simplicidad constructiva, industrial y de uso. Existen multitud de soluciones de gran efectividad que pueden cumplir los imprescindibles y estrictos requisitos económicos de la vivienda de bajo coste

La investigación demuestra que los techos verdes cumplen eficientemente la función de aislamiento térmico y estabilidad térmica, pudiendo ser aplicados a gran escala siempre y cuando se impulse la investigación a nivel local en torno a la composición de sustratos, biodiversidad, costos, etc.

\section{Agradecimientos}

La concepción de esta estructura de investigación y el avance de dichos trabajos ha sido planteado en estrecha colaboración con el programa Prometeo del Senescyt así como con las carreras de Ingeniería Mecánica, Ingeniería Mecatrónica e Ingeniería Civil de la Universidad de las Fuerzas Armadas (ESPE). El financiamiento de la construcción del prototipo ESPE II (E2) ha sido realizado por Luis Velasco y Miguel Ángel Bernabé. La Empresa CRYLAMIT ha colaborado desinteresadamente en la provisión de los vidrios. La Universidad de las Fuerzas Armadas ha colaborado mediante la cesión temporal del emplazamiento para la construcción del prototipo E2.

\section{Bibliografía}

Alamino, Y., Kuchen, E., Gil Rostol, C. y Alonso Frank, A. (2015). Monitoreo de funcionamiento y estrategias de eficiencia energética para el edificio público de Obras Sanitarias Sociedad del Estado, San Juan, Argentina. Revista Hábitat Sustentable, 5

Beraldo A.L. (UPV). (2011). Aprovechamiento de residuos agro-industriales como fuente sostenible de materiales de construcción. CYTED. Disponible en: http://www.apuama.org/wp-content/uploads/2012/06/publicationcap14.pdf

Blackhurst, M., Hendrickson, C., Asce, D. M., Matthews, H. S. y Asce, A. M. (2010). Cost-Effectiveness of Green Roofs

C. Filippín, L. M. (2004). Monitoreo higrotérmico, energético y socio ambiental de una escuela solana en la provincia de la Pampa. Avances en Energías Renovables y Medio Ambiente, 8

Castañeda, G., Argüello, T. y Vecchia, F. (2011). Desempeño Térmico de Techo Alternativo para Vivienda en Tuxtla, México

Heine Leandro, M. G. (2002). Doble fachada en edificios. Conceptos y aplicación para Argentina. Avances En Energías Renovables y Medio Ambiente, 6. Disponible en: http://doi.org/10.1017/CBO9781107415324.004

INAMHI. (n.d.). Anuarios meteorológicos.

Machado, M., Brito, C., y Neila, J. (2000). La Cubierta Ecológica como Material de Construcción. Informes de la Construcción, 52 
estudios del hábitat | Vol. 17 (1) e063 | Junio 2019 ISSN 2422-6483

Neila, F., Bedoya, C. y Acha, C. (2008). Las cubiertas ecológicas de tercera generación : un nuevo material constructivo. Informes de La Construcción, 60(511)

Disponible en: http://informesdelaconstruccion.revistas.csic.es/index.php/informesdelaconstruccion/article/ viewArticle/742

Salas, J. y Veras Castro, J. (2012). Materiales de construcción con propiedades aislantes a base de cascara de arroz. Informes de La Construcción, 37(372). Disponible en: http://doi.org/10.3989/ic.1985.v37.i372.1856

Salazar, J., García, C. D., y Olaya, J. M. (n.d.). Dosificación de hormigones ligeros con cascarilla de café. Ingeniería e Investigación, 51-56.

Velasco, L., Delgado, R. y Goyos, L. (2015). Investigación y desarrollo de aislantes térmicos naturales basados en residuos de biomasa para su aplicación en la mejora de la eficiencia energética de las edificaciones en América Latina. Ingeniería y sociedad, $n^{\circ} 10$ vol.1. 